\title{
La incidencia del Impuesto a la renta en la captación de recursos en las Cooperativas de Ahorro y Crédito en la ciudad de Riobamba - Ecuador, períodos 2012-
} 2016

\section{La incidencia del Impuesto a la renta en la captación de recursos en las Cooperativas de Ahorro y Crédito en la ciudad de Riobamba-Ecuador, períodos 2012-2016}

Jorge Enrique Arias Esparza. ${ }^{1}$

\begin{abstract}
.
The impact that tax collection has on the economy of a country is significant, either for positive aspects such as economic development or restrictive aspects for specific sectors where costs increase and profits decrease. This research presents the incidence in the income contribution on the collection of resources in the Savings and Credit Cooperatives in the city of Riobamba-Ecuador in the period 2016 - 2018, key year for the projections and determinants of new regulations for the following periods. Work carried out through a qualitative-quantitative, retrospective, cross-sectional, documentary, non-experimental methodology in the analytical and synthetic evaluation of statistical data. The population was made up of the Managers, Accountants and administrative personnel of the main Savings and Credit Cooperatives of different segments registered in the Superintendency of Popular and Solidarity Economy, to whom a variable relationship scheme was applied, relying on their reliability aa through the Likert scale. Concluding that it has existed in percentage terms in each of the chosen institutions, recommending better strategies that involve improving the level of fundraising.
\end{abstract}

Keywords: Income tax, collection, popular economy, cooperatives.

\footnotetext{
${ }^{1}$ Universidad Nacional Mayor de San Marcos, Facultad de Ciencias Contables, Doctorado en Ciencias Contables y Empresariales, jorgehenry_66@ hotmail.com, https://orcid.org/ 0000-2290-9043-0009
} 


\section{Resumen.}

La incidencia que tiene la recaudación tributaria para la economía de un país es significativa, ya sea para aspectos positivos como el desarrollo económico o restringentes para sectores específicos donde se incrementan los costos y disminuyen las utilidades. En la presente investigación se presenta la incidencia en la contribución a la renta sobre la captación de recursos en las Cooperativas de Ahorro y Crédito en la ciudad de RiobambaEcuador en el periodo 2012 - 2016, año clave para las proyecciones y determinantes de nueva normativa para los siguientes periodos. Trabajo realizado a través una metodología de tipo cuali-cuantitativa, retrospectiva, transversal, documental, no experimental en la evaluación analítica y sintética de datos estadísticos. La población estuvo conformada por los Gerentes, Contadores y el personal administrativo de las principales Cooperativas de Ahorro y Crédito de distintos segmentos registrados en la Superintendencia de Economía Popular y Solidaria, a quienes se aplicó un esquema de relación de variables, contando con su fiabilidad a través de la escala de Likert. Concluyendo que ha existido porcentualmente en cada una de las instituciones elegidas, recomendando mejores estrategias que involucren mejorar el nivel de captación de recursos.

Palabras claves: Impuesto a la renta, captación, economía popular, cooperativas

\section{Introducción.}

El Estado Ecuatoriano dentro del financiamiento de su presupuesto tiene varios componentes, entre los principales ingresos con los que cuenta son los tributos que se conforman por los impuestos, tasas y contribuciones; los impuestos son generados por el sujeto activo (estado), en tanto que los sujetos pasivos, son todos los contribuyentes (personas naturales o jurídicas); este componente es parte fundamental del financiamiento del presupuesto general del estado; entre los principales impuestos se tiene el Impuesto a la Renta, Impuesto a los Consumos Especiales, el Impuesto a la Salida de Capitales y el Impuesto al Valor Agregado (IVA), existiendo otros de menor jerarquía o recaudación. Definición que se corrobora con el autor Larrea, en su Guía Práctica del Régimen Tributario, "Sujeto activo (entidad acreedora) de la obligación tributaria es, principalmente, el Estado; pero también puede serlo cualquier otra entidad pública facultada al respecto. El sujeto activo, como entidad acreedora, recauda los tributos en la forma y con los requisitos exigidos por la ley. Por otra parte, el Sujeto pasivo de la obligación tributaria, serán las personas que están obligadas a pagar los tributos en la forma y con los requisitos exigidos por la ley (Larrea, 2012, pág. 13).

A su vez, autores como Orellana y Martínez definen a los sujetos pasivos de la obligación tributaria, como las personas que están obligadas a pagar tributos y con los requisitos exigidos por la ley. Nuestra legislación reconoce a contribuyentes y responsables. Siendo las personas responsables aquellas que no tienen la calidad de contribuyente, pues no 
están vinculadas directamente con el hecho generador; para lograr un mejor control en la recaudación de tributos y en razón del cargo o función que esas personas ocupen por disposición expresa de la ley, deben cumplir sus obligaciones asignadas a los contribuyentes. De ahí que los responsables siempre serán sujetos pasivos, pero por deuda ajena (Martínez, 2015, págs. 15-19).

En los últimos años la cultura tributaria en Ecuador ha venido incrementándose a través de campañas que promueven las leyes ejecutables para la recaudación; sin embargo, la incidencia que tiene en los sectores económicos de acuerdo a un análisis específico no ha sido favorable y por el contrario ha recibido críticas a los montos y la dependencia que ha podido adquirir la economía de estas contribuciones.

Un objetivo clave del análisis de la tributación es identificar el o los grupos sobre los que recae el pago de cada impuesto. Del mismo modo es importante observar y cuantificar al grupo de beneficiarios y al grupo de perjudicados de una modificación o reforma tributaria (Salazar, 2013, pág. 45).

En materia tributaria debe distinguirse dos conceptos: transferencia (traslación) e incidencia. La persona que paga originalmente impuestos bien puede no ser, la que, en última instancia, efectivamente esté afecta por dicha carga tributaria.

Por tanto, la incidencia tributaria será un resultado del proceso de transferencia o traslación de impuestos. Cuando hay un proceso de transferencia de impuestos, éste puede ser hacia atrás o hacia adelante (Rodríguez, 2019, págs. 123-125).

La incidencia tributaria definida en términos generales comprende el estudio de los efectos de la política tributaria sobre la distribución del bienestar económico. La colocación de impuestos afecta el precio de los bienes y/o la retribución a los factores de producción. Para evaluar la incidencia tributaria se tiene que conocer los efectos de ésta sobre los distintos mercados y agentes económicos.

La incidencia tributaria comprende varias dimensiones tales como efectos de los impuestos en la distribución del ingreso de los factores, sobre el grado de desigualdad del ingreso, sobre el bienestar intergeneracional y sobre los consumidores de diferentes productos.

Diferentes estudios señalan que las conclusiones que se deriven del análisis de incidencia tributaria dependerán del modelo de equilibrio elegido, además dichos resultados son generalmente ambiguos si no se hacen precisiones sobre la naturaleza de las preferencias y la tecnología de los agentes económicos. En esta investigación la incidencia está dirigida a un sector de crecimiento dimensional dentro de la economía como el sector financiero popular y solidario, el cual acoge a todo un sistema de entidades categorizadas por su cantidad y volumen de activos. Estas entidades son todas las cooperativas de ahorro y crédito.

El sistema cooperativo de ahorro y crédito al estar inmerso dentro de la actividad de intermediación financiera en el contexto nacional, en estos últimos años se ha constituido 
en un canalizador preponderante de recursos a los sectores menos favorecidos con créditos de diferentes tipos, cubriendo una gran parte del mercado de la población de menores ingresos; obteniendo recursos por medio del ahorro y de aportes internacionales que fomentan este tipo de organizaciones de carácter social y solidarias; regentadas por la Superintendencia de Economía Popular y Solidaria.

Con la actualización de leyes, normas, y entidades de control en el país, el acceso a los datos estadísticos de existencia y operación de las entidades cooperativistas han mejorado tal es así que se identifica un gran número de las mencionadas entidades en la provincia de Chimborazo correspondiente al 36\% del total nacional, siendo la cabecera cantonal Riobamba, el lugar de asentamiento mayoritario para oficinas matrices, sucursales y agencias (SEPS, Superintendencia de Economía Popular y Solidaria, 2019).

\section{Metodologia.}

Ramírez (2015) refieren que el tipo de la investigación está determinado por su metodología y los métodos que la constituyan. En cuanto a la metodología, de acuerdo a la naturaleza del problema planteado y objetivos propuestos, la investigación es de tipo descriptiva con un enfoque cuali-cuantitativo. Respecto al método empleado, es decir de acuerdo a la estrategia que se utilizó para comprobar las hipótesis formuladas, responde al tipo transversal y retrospectiva. Asimismo, esta investigación conocida cuantitativa corresponde al tipo analítico, descriptivo, correlacional y no experimental (Ramírez, 2013, págs. 14-15).

El desarrollo de la investigación comprendió la construcción del marco teórico necesario para el adecuado conocimiento e interpretación de las variables identificadas, a fin de evaluar su incidencia en la captación de recursos en las Cooperativas de Ahorro y Crédito en la ciudad de Riobamba - Ecuador período 2012 - 2016. Se realizó una entrevista y se recurrió también a la técnica de la observación.

La unidad de análisis lo constituyeron las Cooperativas de Ahorro y Crédito (COACs) de la ciudad de Riobamba. La población que se consideró en esta fueron los Gerentes y Contadores, personal administrativo de las Cooperativas de Ahorro y Crédito de distintos segmentos registrados en la Superintendencia de Economía Popular y Solidaria hasta el año 2018 (SEPS, Sistema Financiero Popular y Solidario, 2018)

La investigación se basó entre el incremento de la recaudación del impuesto a la renta y la captación de recursos financieros en las Cooperativas de Ahorro y Crédito de la ciudad de Riobamba, se encuentra en el campo no experimental, dado que se observarán los fenómenos, hechos, situaciones o sujetos en su ambiente natural o realidad, y no han sido provocados intencionalmente por el investigador. 


\section{Resultados.}

El resultado principal se manifiesta a través del esquema de métodos utilizados en la investigación y las cifras recabadas para las principales cooperativas

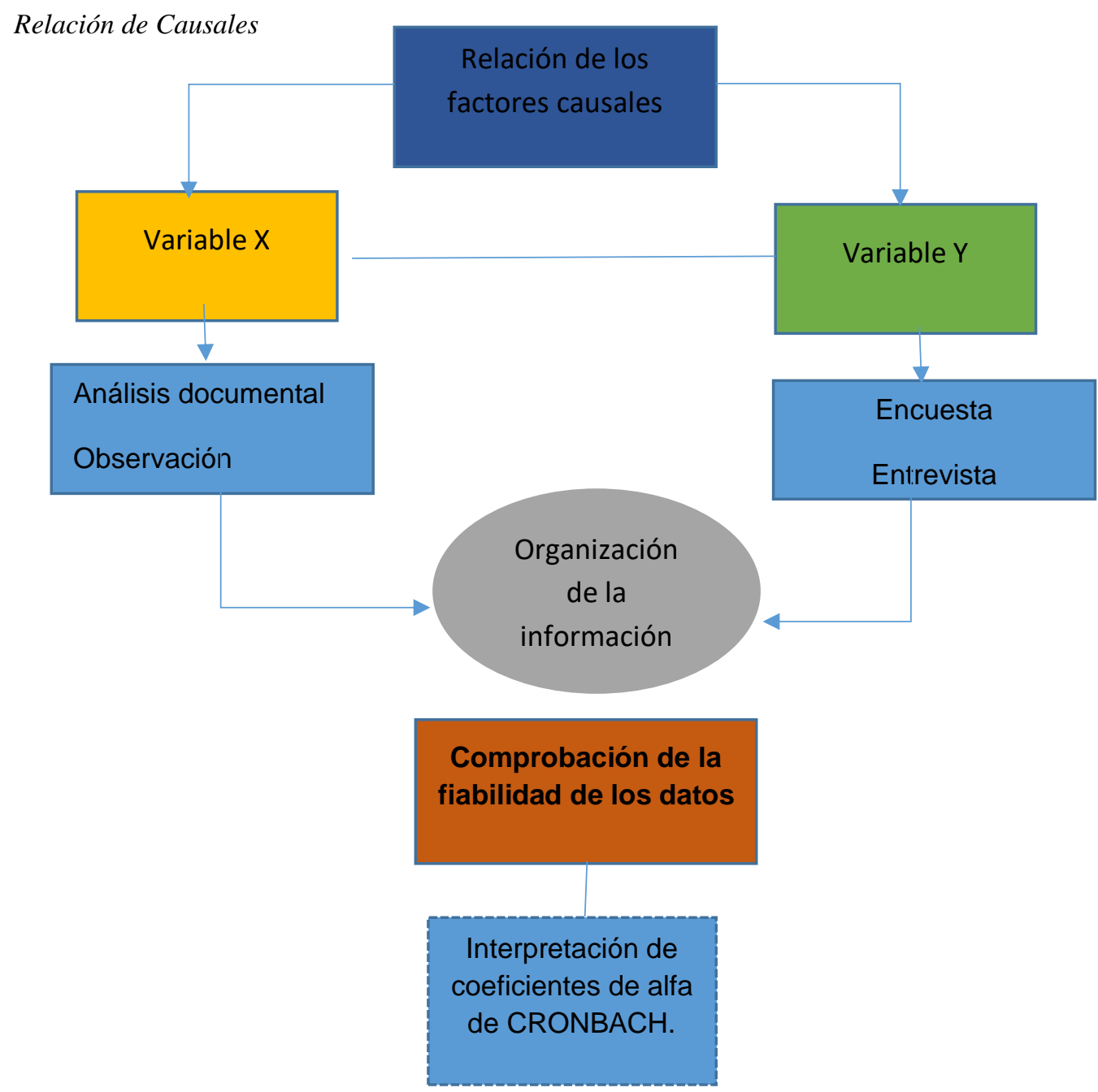

Figura 1.- Esquema de métodos utilizados en la investigación Fuente: Elaboración propia

\section{Relación de los factores causales}

El principal resultado demuestra la relación de los factores causales que relacionan el problema de investigación mediante la correlación entre las variables $(\mathrm{X})$ independiente, con el efecto en la variable (Y) dependiente. 
Tabla 1.-

\section{Relación de variables.}

\begin{tabular}{cll}
\hline X=VI: & $\begin{array}{l}\text { Variable } \\
\text { Independiente } \\
\text { Variable }\end{array}$ & $\begin{array}{l}\text { Impuesto a } \\
\text { la Renta }\end{array}$ \\
X1=VI1: & $\begin{array}{l}\text { Independiente } \\
\text { uno }\end{array}$ & Por niveles \\
& Variable & Por \\
X2=VI2 & $\begin{array}{l}\text { Independiente dos } \\
\text { Variable }\end{array}$ & segmentos \\
& Recursos \\
Y=VD: & Dependiente & Financieros \\
\hline
\end{tabular}

Fuente: Elaboración propia

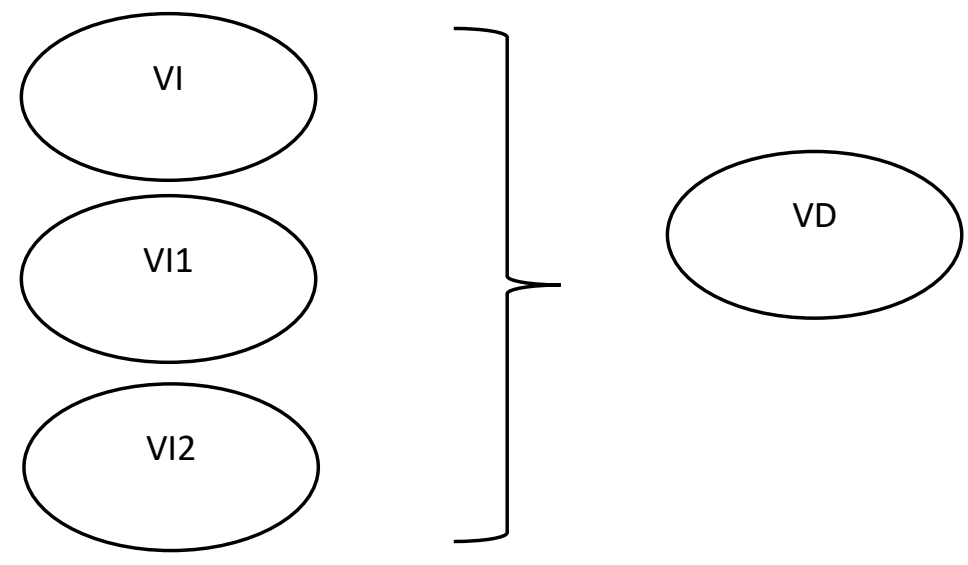

Figura 2.- Relación de variables.

Fuente: Elaboración propia

Para el estudio de la variable (X) "IMPUESTO A LA RENTA" se aplicará: el análisis documental, mediante el cual permite analizar los hechos del pasado relacionados con las declaraciones tributarias realizadas en años anteriores para verificar la evolución del aporte de estas cooperativas al presupuesto del estado por medio de este impuesto; La observación, que permitirá evidenciar datos originales sobre el desarrollo de las prácticas empleadas en los procesos de tributación.

Para el estudio de la variable (y) se aplicará una encuesta, la cual permitirá recabar criterios de cómo generar estrategias eficaces de manera masiva para mejorar los niveles de captación de recursos económicos en el mercado financiero de la ciudad de Riobamba. Técnica que permitirá cuantificar sus resultados, convirtiéndose en indicador para la elaboración de la propuesta. 
Además, que a través de la entrevista se podrá acceder a la parte mental de las personas, pero también a su parte vital a través de la cual se descubrirá la cotidianidad y las relaciones que mantienen las Cooperativas de Ahorro y Crédito en Riobamba.

La pertinencia de la entrevista es alta, ya que contribuye a situar la cuantificación de un contexto social y cultural más amplio. A través de los Instrumentos se extraerá la información; estos serán cuidadosamente elaborados en su forma y contenidos, deberán medir la variable, deben tener validez de contenidos, validez de criterio y validez de constructo y serán objetivos para evitar sesgos. Se utilizarán tablas de cotejo, cuestionarios de entrevistas y encuestas, fichas.

\section{Organización de la información:}

La información recabada se organiza mediante un proceso de tabulación, las preguntas fueron evaluadas con criterios establecidos en la escala de Likert, y se efectuó aplicando el análisis de correlación de Pearson asumiendo un nivel de significación de 0.5 para determinar el nivel de asociación entre las variables consideradas. Se elaboró los gráficos respectivos y los análisis correspondientes.

La utilización de la escala de Likert permite a los encuestados que tengan una mejor comprensión y una amplitud en el momento de escoger una alternativa al requerimiento planteado en la investigación, lo que a su vez aumentó la tasa de respuestas proporcionadas.

\section{Fiabilidad de los datos}

Como criterio general de (Darren George , Paul Mallery, 2003) Pág. 213 sugieren unas recomendaciones para evaluar los valores de los coeficientes del alfa de Cronbach obtenidos durante la investigación.

Tabla 2.-

Interpretación de coeficientes de alfa de cronbach.

\begin{tabular}{ll}
\hline Coeficiente alfa Mayor que 9 & EXCELENTE \\
Coeficiente alfa mayor que 8 & BUENO \\
Coeficiente alfa mayor que 7 & ACEPTABLE \\
Coeficiente alfa mayor que 6 & CUESTIONABLE \\
Coeficiente alfa mayor que 5 & POBRE \\
Coeficiente alfa MENOR que 5 & INACEPTABLE \\
\hline \multicolumn{2}{c}{ Fuente: George y Mallery } \\
\hline
\end{tabular}


Tabla 3.-

Estadísticas de Fiabilidad

\begin{tabular}{|c|c|c|}
\hline $\begin{array}{c}\text { Alfa de } \\
\text { Cronbach }\end{array}$ & $\begin{array}{c}\text { Alfa de } \\
\text { Cronbach } \\
\text { basada en } \\
\text { elementos } \\
\text { estandarizados }\end{array}$ & $\mathrm{N}$ de elementos \\
\hline ,869 & ,840 & 13 \\
\hline
\end{tabular}

La fiabilidad de los datos recolectados es de vital importancia ya que indica la consistencia de los datos mediante la correlación entre ellos. En el presente trabajo investigativo, el nivel de confiabilidad se procedió a calcular el coeficiente Alfa de Cronbach; cuyo resultado que se ve reflejado en la tabla 3 y asciende a 0,869 lo que indica que el instrumento con que se recolectó los datos tiene un nivel confiabilidad BUENO según de la escala presentada en la tabla 2.

Nivel de Captación de las Cooperativas de Ahorro y Crédito

Tabla 4.- \% Captación del Mercado

\begin{tabular}{cc}
\hline COAC's & $\begin{array}{c}\text { \% Captación del } \\
\text { Mercado }\end{array}$ \\
\hline Coop. De Ahorro y Crédito Riobamba & $17 \%$ \\
\hline Coop. De Ahorro y Crédito Oscus & $11 \%$ \\
\hline Coop. De Ahorro y Crédito Sagrario & $12 \%$ \\
\hline Coop. De Ahorro y Crédito Minga & $6 \%$ \\
\hline Coop. De Ahorro y Crédito Daquilema & $7 \%$ \\
\hline Coop. De Ahorro y Crédito Chibuleo & $9 \%$ \\
\hline Coop. De Ahorro y Crédito CRECER WIÑARI & $4 \%$ \\
\hline Coop. De Ahorro y Crédito CACPECO & $13 \%$ \\
\hline Coop. De Ahorro y Crédito Educadores De Chimborazo & $9 \%$ \\
\hline Coop. De Ahorro y Crédito Policía Nacional & $12 \%$
\end{tabular}

Fuente: Boletines Anuales SEPS

Nivel de Impuesto o a la Renta

Tabla 5.- \% Impuesto a la Renta

\begin{tabular}{ccccccc}
\hline COAC's & $\mathbf{2 0 1 2}$ & $\mathbf{2 0 1 3}$ & $\mathbf{2 0 1 4}$ & $\mathbf{2 0 1 5}$ & $\mathbf{2 0 1 6}$ & $\begin{array}{c}\text { Porcentaje de } \\
\text { Incremento }\end{array}$ \\
\hline $\begin{array}{c}\text { Coop. De } \\
\text { Ahorro y } \\
\text { Crédito } \\
\text { Riobamba }\end{array}$ & $\$ 2,358,834.21$ & $\$ 1,653,100.24$ & $\$ 1,031,245.18$ & $\$ 1,111,982.63$ & $1,111,982.63$ & $2 \%$ \\
\hline $\begin{array}{c}\text { Coop. De } \\
\text { Ahorro y } \\
\text { Crédito Oscus }\end{array}$ & $\$ 2,113,255.09$ & $\$ 2,393,924.50$ & $\$ 1,718,586.11$ & $\$ 591,837.09$ & $1,111,982.63$ & $-37 \%$ \\
\hline
\end{tabular}


ISSN: 2602-8506

Vol. 5, $\mathrm{N}^{\circ}$ 2, p. 106-118, abril-junio, 2021

\begin{tabular}{|c|c|c|c|c|c|c|}
\hline $\begin{array}{c}\text { Coop. De } \\
\text { Ahorro y } \\
\text { Crédito } \\
\text { Sagrario }\end{array}$ & $\$ 1,227,775.85$ & $\$ 1,113,586.01$ & $\$ 719,423.81$ & $\$ 578,603.91$ & $1,111,982.63$ & $-20 \%$ \\
\hline $\begin{array}{c}\text { Coop. De } \\
\text { Ahorro y } \\
\text { Crédito } \\
\text { Minga }\end{array}$ & $\$ 38,142.30$ & $\$ 21,967.66$ & $\$ 0.00$ & $\$ 28,010.56$ & $1,111,982.63$ & $15 \%$ \\
\hline $\begin{array}{c}\text { Coop. De } \\
\text { Ahorro y } \\
\text { Crédito } \\
\text { Daquilema }\end{array}$ & $\$ 858,149.38$ & $\$ 695,073.82$ & $\$ 245,210.35$ & $\$ 92,702.57$ & $1,111,982.63$ & $-56 \%$ \\
\hline $\begin{array}{l}\text { Coop. De } \\
\text { Ahorro y } \\
\text { Crédito } \\
\text { Chibuleo }\end{array}$ & $\$ 627,602.95$ & $\$ 450,355.93$ & $\$ 117,774.62$ & $\$ 76,337.31$ & $1,111,982.63$ & $-42 \%$ \\
\hline $\begin{array}{c}\text { Coop. De } \\
\text { Ahorro y } \\
\text { Crédito } \\
\text { CRECER } \\
\text { WIÑARI }\end{array}$ & $\$ 1,855.78$ & $\$ 3,383.23$ & $\$ 2,740.21$ & $\$ 30,303.00$ & $1,111,982.63$ & $-65 \%$ \\
\hline $\begin{array}{c}\text { Coop. De } \\
\text { Ahorro y } \\
\text { Crédito } \\
\text { CACPECO }\end{array}$ & $\$ 2,970,956.30$ & $\$ 2,568,912.31$ & $\$ 1,878,920.93$ & $\$ 1,250,147.10$ & $1,111,982.63$ & $-12 \%$ \\
\hline $\begin{array}{c}\text { Coop. De } \\
\text { Ahorro y } \\
\text { Crédito } \\
\text { Educadores } \\
\text { De } \\
\text { Chimborazo }\end{array}$ & $\$ 137,074.49$ & $\$ 83,117.43$ & $\$ 70,362.85$ & $\$ 87,958.31$ & $1,111,982.63$ & $13 \%$ \\
\hline $\begin{array}{c}\text { Coop. De } \\
\text { Ahorro y } \\
\text { Crédito } \\
\text { Policía } \\
\text { Nacional }\end{array}$ & $\$ 2,380,913.81$ & $\$ 2,818,701.18$ & $\$ 1,948,125.04$ & $\$ 846,862.27$ & $1,111,982.63$ & $-14 \%$ \\
\hline
\end{tabular}

Fuente: Servicio de Rentas Internas (2018)

\section{Discusión}

Las cooperativas son asociaciones voluntarias de personas y no de capitales, con plena personalidad jurídica, de duración indefinida y de responsabilidad limitada, en la que los individuos se organizan democráticamente a fin de satisfacer sus necesidades y promover su mejoramiento económico y social, como un medio de superar su condición humana y su formación individual, y en las cuales el motivo del trabajo y la producción, de la distribución y del consumo, es el servicio y no el lucro (Cooperativas de Costa Rica, 2019). “... es una asociación autónoma de personas que se han unido voluntariamente para hacer frente a sus necesidades y aspiraciones económicas, sociales y culturales comunes, por medio de una empresa de propiedad conjunta y democráticamente controlada..." (Declaración de Manchester., 1995). Al conocer estas definiciones que parten de la doctrina cooperativista es difícil creer cómo la incidencia del Impuesto a la renta en la captación de recursos en este tipo de entidades haga parte de un análisis que se haría o reflejaría a entidades de intermediación con fines de lucro; partiendo de que el lucro según el autor Guillermo Cabanellas es la ganancia, provecho, utilidad o beneficio 
que se obtiene de alguna cosa (Torres, 2019). Por lo que, la doctrina estaría infringida por una norma. A la vez, Normas que están emitidas por entidades de control y la misma política económica de un gobierno.

El cooperativismo como doctrina es el conjunto de principios, valores y normas que regulan y orientan el comportamiento y las actividades de los individuos dentro de una organización cooperativa. La doctrina cooperativa se basa en tres grandes conceptos: a) La Igualdad b) La Libertad c) La Solidaridad.

Sin embargo, cuando a normas y leyes emitidas por un país o un Estado de Derecho la pregunta es saber cómo se dictan y si estas pueden suprimir doctrinas legítimas sobre la cual están constituidas organizaciones como las cooperativas. Puede ser que en este último esté la razón del lugar que ocupa el país en la historia cooperativista de crecimiento en el mundo.

Las cooperativas de ahorro y créditos nacen en Alemania. Luego, a finales de ese mismo siglo, el cooperativismo llegó a Latinoamérica con los torrentes de inmigrantes europeos a Argentina, Uruguay y Sur de Brasil. En ese momento el movimiento se bifurcaba en dos grandes tendencias ideológicas. Una, la utópica representada por Charles Gide y su "República Cooperativa". Otra, la pragmática, que se fundamentaba en las cajas Raiffeisen de Alemania y en la herencia de la cooperativa de Rochadle, modelos estos que buscaban mejorar las condiciones de vida de los trabajadores a través de cooperativas de consumo, ahorro y crédito. El grupo más desarrollado en lo que a cooperativismo se refiere está constituido por los países del sur: Argentina, Uruguay, Sur de Brasil y Chile. En este último, el cooperativismo fue desarticulado durante la dictadura del ex presidente, Augusto Pinochet. El segundo grupo está conformado por: México, Costa Rica y Puerto Rico, cuyo cooperativismo consiguió ciertos niveles de crecimiento. El tercer grupo está integrado por los países Andinos: Colombia, Venezuela, Perú, Ecuador y Bolivia. Estos, aparte de los centroamericanos, exceptuando a Costa Rica y los países del Caribe, con omisión de Puerto Rico. En estos países el cooperativismo llegó a partir de 1930 por lo que se hace un sistema aún nuevo, además de que, básicamente se ha limitado al ahorro y crédito y al consumo como áreas de producción (Doctrina Cooperativa, 2019).

Razones que denotan, la incipiente actividad y la carga impositiva que adquieren por sus captaciones. Por otra parte, el Estado sólo contrasta con un beneficio que ofrece la legislación ecuatoriana y se encuentra detallado en los artículos 9 y 37 de la Ley Orgánica de Régimen Tributario Interno es la Reinversión de Utilidades, que versa sobre la reducción de puntos porcentuales en la tarifa del Impuesto a la Renta de Sociedades sobre la utilidad a reinvertir, siempre y cuando esos recursos sean destinados a incrementar el rendimiento y empleo mediante la adquisición de Activos fijos productivos o bienes relacionados con la investigación y tecnología que genere diversificación. La vez, que podrían ser solidariamente repartidos entre sus socios. 
Además de lo expuesto se debe considerar que la progresión del Impuesto a la Renta no se aplica para las sociedades legalmente constituidas en el país, las sucursales y establecimientos permanentes de sociedades extranjeras no domiciliadas, para éstas existe una tarifa única, que hasta el año 2010 era del 25\% sobre la base imponible. Ésta tarifa ha tenido modificaciones desde la aparición del Código Orgánico de la Producción, Comercio e Inversiones que dispuso para el año 2011 una tarifa del 24\%, para el año 2012 de $23 \%$ y para el año 2013 en adelante una tarifa del $22 \%$.

Por otra parte, las sociedades que reinviertan sus utilidades en el país tendrán una deducción del $10 \%$ sobre las utilidades que reinviertan y sobre la parte que no lo hagan pagarán el porcentaje previsto para sociedades. Para poder acceder a este beneficio las sociedades deberán realizar el aumento de capital por el valor de las utilidades reinvertidas e inscribir el mismo en el Registro Mercantil hasta el 31 de diciembre del Ejercicio Impositivo siguiente. El monto reinvertido debe ser empleado en la adquisición de activos productivos, que se utilicen para su actividad productiva, generen diversificación productiva e incremento de empleo. Para las organizaciones de Economía Social y Solidaria también se aplica este beneficio siempre y cuando lo destinen al otorgamiento de créditos.

\section{Conclusiones.}

- La recaudación de impuestos para un Estado es de vital importancia, de ahí que se puedan lograr recursos para cumplir con presupuestos de obras, salud, educación; sin embargo, son estos recursos los que pueden generar en las empresas u organizaciones del Sistema Financiero Popular, disminuir sus utilidades o afectar directamente en su giro de negocio, entendido como un negocio solidario, donde el ánimo no es el lucro sino el fortalecimiento de los sectores no bancarizados.

- En los datos obtenidos varias de estas organizaciones presentan un menor nivel de captación, lo que se traduce en menores recursos para la colocación, y por ende el incremento en el valor de los mismos, contemplando este valor en la referencial tasa de interés de su utilización. Por lo que, es imprescindible acogerse a los beneficios directos que se ofrece para este tipo de organizaciones.

\section{Bibliografía}

Cooperativas de Costa Rica. (2019). Ley de Asociaciones Cooperativas de Costa.

Darren George , Paul Mallery. (2003). SPSS para Windows Paso a Paso: una guía sencilla y referencia, actualización 11.0. Michigan: ISBN 0205375529, 9780205375523.

Declaración de Manchester. (1995). Declaración de Manchester., A.C.I. . 
Doctrina Cooperativa. (Mayo de 2019). Obtenido de Doctrina Cooperativa: http://www.incoop.gov.py/v2/wp-content/uploads/2011/06/DoctrinaCooperativa.pdf

Larrea, L. (2012). Tributos, Contribuciones para el Estado. Recuperado el 25 de Mayo de 2019, de Tributos, Contribuciones para el Estado.

Martínez, O. \&. (2015). Tributación y Definiciones. Santiago, Chile: Brander.

Ramírez, P. (2013). Metodología de la Invetigación. Bogotá: PRANDIZ.

Rodríguez, M. (1 de Junio de 2019). Incidencia Tributaria. Obtenido de Incidencia Tributaria: http://www.incidencia_tributaria_etudio_caso.com

Salazar, E. (2013). Cambios del Sistema Tributario. Recuperado el Mayo de 2019, de www.contabilidad_tributos_tax.com

SEPS. (2018). Sistema Financiero Popular y Solidario. Boletín SEPS, 15-24.

SEPS. (30 de Mayo de 2019). Superintendencia de Economía Popular y Solidaria. Obtenido de www.seps.gob.ec

Torres, G. C. (2019). Diccionario Juríico. Obtenido de Lucro: https://www.google.com/search?client=firefox-b-

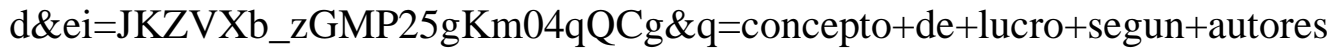
\&oq=lucro+segun+au\&gs_l=psyab.1.1.0i22i3012.1941567.1949096..1953116...1.0..1.1822.11502.0j3j1j1j61j1j5......0....1..gws-wiz.......0i71j0i131j0j3

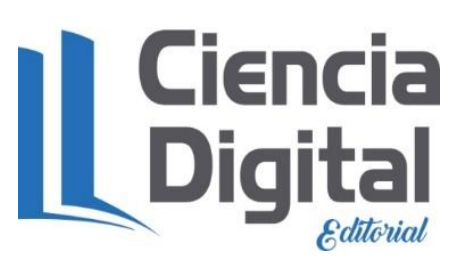




\section{PARA CITAR EL ARTÍCULO INDEXADO.}

Enrique Arias, J. (2021). La incidencia del Impuesto a la renta en la captación de recursos en las Cooperativas de Ahorro y Crédito en la ciudad de Riobamba - Ecuador, períodos 2012-2016. Visionario Digital, $\quad$ 5(2), 106-118. https://doi.org/10.33262/visionariodigital.v5i2.1661

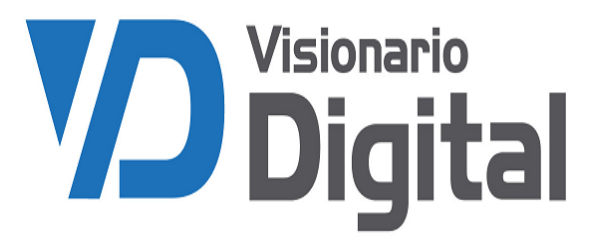

El artículo que se publica es de exclusiva responsabilidad de los autores y no necesariamente reflejan el pensamiento de la Revista Visionario Digital.

El artículo queda en propiedad de la revista y, por tanto, su publicación parcial y/o total en otro medio tiene que ser autorizado por el director de la Revista Visionario Digital.
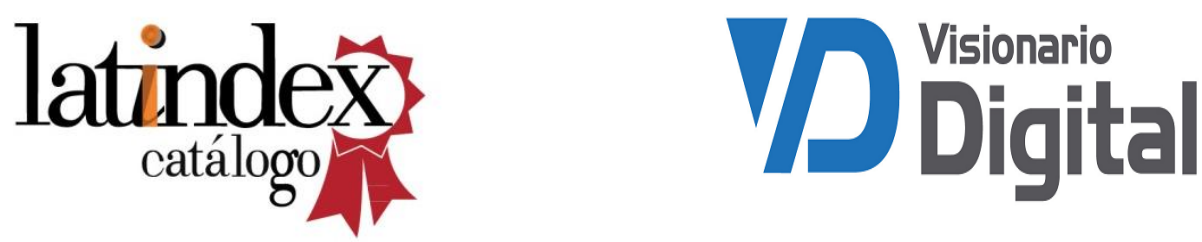